Tropical Journal of Pharmaceutical Research October 2012; 11 (5): 835-845

(C) Pharmacotherapy Group,

Faculty of Pharmacy, University of Benin

Benin City, 300001 Nigeria.

All rights reserved.

Available online at http://www.tjpr.org http://dx.doi.org/10.4314/tjpr.v11i5.18

Research Article

\title{
Intermittent Preventive Therapy and Treatment of Malaria during Pregnancy: A Study of Knowledge among Pregnant Women in Rufiji District, Southern Tanzania
}

\author{
Ritah Mutagonda ${ }^{1}$, Appolinary AR Kamuhabwa ${ }^{1 *}$, Siriel Massawe ${ }^{2}$ and \\ Rose Mpembeni ${ }^{3}$ \\ ${ }^{1}$ Unit of Pharmacology and Therapeutics, School of Pharmacy, ${ }^{2}$ Department of Obstetrics and Gynaecology, School \\ of Medicine, ${ }^{3}$ Department of Epidemiology and Biostatistics, School of Public Health and Social Sciences, Muhimbili \\ University of Health and Allied Sciences, Dar es Salaam, Tanzania
}

\begin{abstract}
Purpose: To assess the knowledge and awareness of pregnant women regarding the use of sulfadoxinepyrimethamine (SP) for intermittent preventive therapy (IPT) and artemether-lumefantrine (ALu) for treatment of malaria during pregnancy.

Methods: The study was conducted in Rufiji district, southern Tanzania from March 2011 to September 2011. Four hundred and seventy (470) pregnant women in their second and third trimesters were interviewed when attending antenatal clinics at the selected hospitals, health centers and dispensaries. Focus group discussions (FGDs) were also conducted with 46 pregnant women at the health facilities in the district.

Results: More than half (54.3\%) of pregnant women did not know if SP it was used for IPT. Most women (76.6\%) did not know the use of SP for IPT in relationship with gestation age. Overall, the results show that most women had very low knowledge about the use of SP for IPT. Forty three (9.1\%) pregnant women reported to have had malaria during their current pregnancies. The antimalarials reported to be used by pregnant women were quinine $18(42.9 \%)$, SP (23.8 \%), ALu (21.4\%) and sulphamethoxyprazinepyrimethamine (2.4\%). Irrespective of the gestation age of pregnancy, almost all (98.3\%) pregnant women perceived ALu as unsafe drug to be used during pregnancy.

Conclusion: Most pregnant women had minimum knowledge about the use and benefits of SP for IPT and ALu for treatment of malaria during pregnancy. Some erroneous beliefs about the safety of ALu during pregnancy were also identified among pregnant women. For effective implementation of IPT policy and treatment of malaria during pregnancy, pregnant women should be sensitized and educated on the use and benefits of antimalarial drugs.
\end{abstract}

Keywords: Artemether-lumefantrine, Antenatal clinic, Knowledge, Pregnant women, Sulfadoxinepyrimethamine

*Corresponding author: Email: akamuhabwa@muhas.ac.tz, apporug@yahoo.com; Tel: +255 757576985 (mobile), +255222150748 (office); Fax: +25522 2150465 


\section{INTRODUCTION}

Treatment with artemisinin combination therapies (ACTs) in the first trimester is not recommended because of concerns raised by animal experiments which suggested that artemisinin and its derivatives might be teratogenic and cause foetal resorption if given to experimental animals during a narrow time window in early gestation. Studies have confirmed embryotoxic effects of artemisinin and its derivatives in animals, including primates, with risk being confined to a defined period of gestation [1]. The teratogenic effect is thought to involve red blood cells production (erythropoiesis), which implies the human sensitive period would be within the first trimester of pregnancy [2]. Compared to other previously used antimalarial drugs such as chloroquine and sulfadoxine-pyrimethamine (SP), ALu is also perceived by some people as unsafe antimalarial drug to be used by pregnant women [3]. ALu has a complicated 3-days dosage regimen given at the intervals of $12 \mathrm{~h}$ after the first two loading doses, a factor that may lead to non-adherence to the full dose of the drug by patients [4].

SP administered in the $2^{\text {nd }}$ and $3^{\text {rd }}$ trimesters has consistently been shown to be safe and effective for protection against peripheral and placental infection and anemia during pregnancy and as well as reducing the incidence of low birth weight [4]. Women in areas with stable, endemic malaria transmission should receive two to three doses of SP as intermittent prevent therapy (IPT). WHO recommends at least 2 doses of sulfadoxine $(500 \mathrm{mg})$ and pyrimethamine (25mg) to be given to pregnant women after fetal quickening with each dose given not less than one month apart, and all given prior to the last month of pregnancy [5]. In Tanzania, IPT policy for pregnant women was adopted since August 2001 whereby it is recommended to give two doses of SP for IPT. The first dose is given during the $20^{\text {th }}$ week of pregnancy and the second dose is given between the $30^{\text {th }}$ and $36^{\text {th }}$ week under a directly observed therapy.
A number of challenges which can either be contributed by pregnant women, health care providers or poor health care system are likely to affect the effective implementation of using SP for IPT under direct observed therapy at the health facilities. These may include unreliable supply of free SP at the antenatal clinics and shortage of clean and safe water at the health facility. Due to lack of clean and safe water at the health facility, in some cases pregnant women are given SP to be taken at home. This obviously does not guarantee adherence to treatment, and it would have been better if these pregnant women would be asked to bring clean water at the antenatal clinics to ensure that $\mathrm{SP}$ is taken under direct observed therapy [6]. Poor quality of health care services, shortage of staff and the long distances to antenatal clinics are also contributing factors for poor attendance of pregnant women at the antenatal clinics.

Following antimalarial policy change in Tanzania it was reported that coverage for the use of SP was declining, whereby the first dose of IPT with SP in 2005 was $71 \%$ and declined to $65 \%$ in 2007 . The second dose coverage was $38 \%$ in 2005 , but declined to $30 \%$ in 2007 [7]. For effective implementation of IPT policy it is therefore important to encourage pregnant women to regularly attend antenatal clinics. In addition, health care providers at the antenatal clinics should provide adequate information about the use and benefits of SP for IPT. Pregnant women should therefore be informed about the standard dosage of SP and the need to complete the prescribed courses. Pregnant women should also be counseled about unnecessary fear of SP-induced side effects including Steven-Johnson Syndrome [6].

This study was designed to assess the level of knowledge and the acceptance of pregnant women regarding the use of ALu as the drug of choice for treatment of uncomplicated malaria during pregnancy, and the continued use of SP for IPT after it has been replaced by ALu as the drug of 
choice for treatment of malaria. In addition, pregnant women-related factors and other factors that may hinder effective implementation of IPT using SP and malaria treatment in pregnancy using ALu were assessed.

\section{METHODS}

\section{Study site}

The study was conducted from March 2010 to September 2011 in Rufiji district. Rufiji district lies in southern Tanzania about 178 $\mathrm{km}$ south of Dar-es-Salaam, the country's commercial capital and the biggest city in the country. According to the Rufiji demographic surveillance studies in 2008, the district had a population of 47,935 females of child-bearing age.

Rufiji was selected as the study site based on the routine health records collected across the country regarding information on disease patterns for the purpose of health policy planning and monitoring. The records show that the district has one of the highest rates of outpatient consultations for malaria in the country (826 diagnoses per annum per 1,000 populations), hence a malaria endemic area [8]. It is for this reason Rufiji was selected as the study site, also the area being largely rural with a peri-urban environment it can be used to represent rural and urban areas in Tanzania. The population of Rufiji is clustered around Utete (District headquarters), Ikwiriri, Kibiti and Bungu townships. The district has a total of 62 health facilities, including 2 hospitals, 5 health centers and 55 dispensaries. Of the two hospitals one is a district hospital located at Utete town (the district headquarter) and the other is Mchukwi Mission Hospital run by the Pentecost Church of Tanzania.

\section{Study design, population and sampling}

The design of the study was cross sectional which employed both quantitative and qualitative research methods. The study population was pregnant women attending antenatal clinics. Cluster sampling was used for selection of health facilities. The health facilities were divided into three clusters, including hospitals, health centers and dispensaries. All hospitals and health centers in the district were included in the study. A random sample of 8 dispensaries was selected using simple random sampling. Study participants comprised of 470 pregnant women who were attending antenatal clinics during the time of interviews. In addition, 46 pregnant women were involved in the focus group discussions (FGDs).

\section{Data collection process}

\section{Interviews}

Data collection was mainly carried out by the researchers with the help of four trained nurses working at the antennal clinics in Rufiji. A Semi-structured questionnaire written in Kiswahili was used to assess the level of knowledge of pregnant women regarding the use of SP for IPT and ALu for treatment of malaria during pregnancy. Regarding IPT policy, the main focus was on benefits of using SP during pregnancy, dosage regimen, rationale for taking SP under observed treatment therapy and the importance of early attendance to the antenatal clinics. Views on the efficacy and safety of SP use to the unborn baby and the mother were also sought from pregnant women.

Regarding use of ALu for treatment of malaria, the focus was on the awareness and acceptance for its use as the antimalarial drug of choice for pregnant women after the first trimester. In addition, dosage schedule and necessity of meals before ALu intake with special focus on the use of fatty meals when using ALu to increase drug absorption were also assessed. The perceptions of pregnant women regarding multiple doses of $A L u$, its efficacy and safety to the unborn baby were also sought. 
A knowledge scale was developed and used to assess the level of knowledge of pregnant women regarding the use of SP for IPT. The scale was based on six questions that were designed to assess the knowledge of pregnant women for timing and correct use of SP for IPT. One point was awarded for a correct answer and zero point for a wrong answer. Pregnant women's knowledge was then assessed as very low (0 - 1 score), medium ( $2-3$ score) and high ( $4-6$ score).

\section{Focus group discussions}

Focus group discussions (FGDs) were conducted by a social scientist and the researchers using prepared guided format containing probes based on the objectives of the study. Four FGDs were conducted, two at the hospitals and the other two at the health centers. There were at least ten participants in each focus group. Participants were pregnant women attending antenatal clinics at the health centers and hospitals. Selection of participants took into account a mix of pregnant women with different sociodemographic characteristics that were regarded to be important determinants of pregnant women's knowledge and awareness regarding the use of SP and ALu for treatment of malaria and IPT, respectively. These included level of education, current trimester, gravida, marital status and occupation. The main themes for FGDs were on the importance of early antenatal attendance, the use of SP for IPT, the use of $\mathrm{ALu}$ for malaria treatment in pregnant women and other pregnant womenrelated factors that are likely to affect implementation of IPT policy using SP and ALu for malaria treatment.

\section{Data analysis}

Data were coded and entered in the computer for analysis using statistical package for social sciences (SPSS) program version 16, and microsoft excel was used for analysing of multi-response data. Chi-square test was used to compare the knowledge of pregnant women against social demographic factors such as marital status, age, gravid, education level and occupation. The level of knowledge of pregnant women was also tested against the attendance of pregnant women at the antenatal clinics. A $p$-value of < 0.05 was regarded as significant association between the dependent and independent variables.

\section{Ethical consideration}

Permission to conduct the study at the selected health facilities was obtained from Rufiji district medical officer in-charge. The study was granted ethical clearance from Muhimbili University of Health and Allied Sciences Research and Publications Committee. Oral informed consent was solicited in advance of data collection. Study participants were requested to participate and asked for their consent following an explanation about the purpose of the study.

\section{RESULTS}

Out of the 470 women who were interviewed, $227(48.3 \%)$ were in the second trimester and $243(51.7 \%)$ were in the third trimester of pregnancy. More than half $258(54.9 \%)$ of these women were multigravidae who have had 3 or more pregnancies at the time of interview. Most $273(58.1 \%)$ of the participants were in the age group of $20-35$ years, and $58.1 \%$ of all women had attained formal education. Two hundred and thirty six $(50.2 \%)$ respondents were self-employed (farmers, pastoralists and petty business), and $229(48.7 \%)$ were housewives (Table 1).

\section{Knowledge of pregnant women regarding the use of SP for IPT}

Only $110(23.4 \%)$ of pregnant women correctly understood that early antenatal clinic attendance enables women to get the recommended courses of SP for IPT on time. Others reported health checkup (42.6\%), counseling (17.7\%), vaccination (2.8\%) and treatment of various health conditions 
$(13.6 \%)$ as the benefits of early antenatal clinic attendance.

Table 1: Socio-demographic characteristics of pregnant women who were recruited in the study $(\mathrm{N}=470)$

\begin{tabular}{|c|c|c|}
\hline $\begin{array}{l}\text { Socio-demographic } \\
\text { characteristic }\end{array}$ & $\mathbf{N}$ & $\%$ \\
\hline \multicolumn{3}{|l|}{ Age group (years) } \\
\hline$<20$ & 107 & 22.8 \\
\hline $20-35$ & 312 & 66.4 \\
\hline$>36$ & 51 & 10.9 \\
\hline \multicolumn{3}{|l|}{ Education } \\
\hline No formal education & 197 & 41.9 \\
\hline $\begin{array}{l}\text { Not completed primary } \\
\text { education }\end{array}$ & 60 & 12.8 \\
\hline $\begin{array}{l}\text { Completed } \\
\text { education }\end{array}$ & 196 & 41.7 \\
\hline More than primary education & 17 & 3.6 \\
\hline \multicolumn{3}{|l|}{ Current trimester } \\
\hline Second & 227 & 48.3 \\
\hline Third & 243 & 51.7 \\
\hline \multicolumn{3}{|l|}{ Gravida } \\
\hline First pregnancy & 120 & 25.5 \\
\hline Second pregnancy & 92 & 19.6 \\
\hline Third or more pregnancy & 258 & 54.9 \\
\hline \multicolumn{3}{|l|}{ Marital status } \\
\hline Married & 370 & 78.7 \\
\hline Unmarried & 92 & 19.6 \\
\hline Cohabiting/Divorced & 8 & 1.7 \\
\hline \multicolumn{3}{|l|}{ Occupation } \\
\hline House wife & 229 & 48.7 \\
\hline Self employed & 236 & 50.2 \\
\hline Employed & 5 & 1.1 \\
\hline
\end{tabular}

Pregnant women were also interviewed about their awareness of the different methods that have been advocated for prevention of malaria in pregnant women.

A hundred and fifty one $(32.1 \%)$ pregnant women mentioned the use of SP for IPT as one of the methods for protecting pregnant women from malaria. Others mentioned untreated bed nets $(31.1 \%)$, insecticidetreated bed nets $(36.6 \%)$ and indoor spraying with insecticides $(0.2 \%)$. Although 251 (53.3\%) pregnant women reported to have used SP when visiting antenatal clinics, most women did not know the advantages of using SP when pregnant. Two hundred and fourteen $(45.5 \%)$ pregnant women were aware that SP is given for IPT purpose. As an indication that pregnant women did not know why they were given SP, one of the participants in the focus group at Mohoro health centre said:

"We were given three tablets and we were told to swallow them in front of the nurse, but we do not know why we were given those tablets."

In terms of the number of SP tablets to be taken for IPT, 245 (52.1\%) pregnant women mentioned that 3 tablets should be taken at once, $4(0.9 \%)$ mentioned 2 tablets and the rest $221(47 \%)$ did not know how many tables should be taken. It was only 91 (19.4 $\%)$ pregnant women who knew that 2 doses of SP are given for IPT, whereas $220(46.8$ $\%)$ did not know and the rest 158 (33.6\%) said that one dose of SP should be given. Only $80(17 \%)$ pregnant women mentioned that $S P$ is given during the $2^{\text {nd }}$ and $3^{\text {rd }}$ trimesters, although they were also unable to indicate the exact weeks during which SP should be taken. Others did not know the correct timing of SP, with some women mentioning only the $2^{\text {nd }}$ trimester $(29.6 \%)$, third trimester $(6 \%)$, first trimester $(0.2 \%)$, and all the three trimesters of pregnancy $(0.2$ $\%)$. The rest, 221 (47\%) pregnant women were unable to mention the trimester during which SP should be provided. With regard to direct observation therapy for SP, 232 (49.4 $\%)$ pregnant women mentioned that SP is taken under direct observation therapy to improve compliance, 221 (47 \%) did not know and 17 (3.6\%) indicated that it is taken at home.

According to the knowledge scale based on the range of the answers provided by pregnant women for the correct use of SP for IPT, it is shown that $210(44.7 \%)$ pregnant women had very low knowledge, 117 (24.9 $\%)$ had moderate knowledge and 143 (30.4 $\%)$ had high level of knowledge. The level of knowledge regarding use of SP for IPT correlated with age $(p=0.005)$, gravida $(p=$ 
Table 2: The levels of knowledge about IPT policy using SP with respect to marital status, age and gravida of pregnant women $(\mathrm{N}=470)$

\begin{tabular}{llll}
\hline Characteristic & \multicolumn{3}{c}{ Knowledge level } \\
\cline { 2 - 4 } & Very low & Medium & High \\
\hline Marital status & & & \\
Single & $177(37.7 \%)$ & $77(16.4 \%)$ & $116(24.7 \%)$ \\
$\quad$ Married & $31(6.6 \%)$ & $35(7.4 \%)$ & $26(5.5 \%)$ \\
$\quad$ Co-habiting/widowed & $2(0.41 \%)$ & $5(1.1 \%)$ & $1(0.2 \%)$ \\
Age & & & \\
$\quad<30$ years & $158(33.6 \%)$ & $91(19.4 \%)$ & $88(18.7 \%)$ \\
$\quad>30$ 's years & $52(11.1 \%)$ & $26(5.5 \%)$ & $55(11.7 \%)$ \\
Gravida & & & \\
$\quad \begin{array}{lll}\text { Primigravida } \\
\text { Multigravida }\end{array}$ & $51(10.9 \%)$ & $40(8.5 \%)$ & $29(6.2 \%)$ \\
\hline
\end{tabular}

Table 3: Frequency of attendance to the antenatal clinics as reported by pregnant women during interviews $(\mathrm{N}=470)$

\begin{tabular}{lcc}
\hline Variable & $\mathbf{N}$ & $\%$ \\
\hline Number of visits to antenatal clinics & & \\
First & 141 & 30.0 \\
Second & 179 & 38.1 \\
Third & 105 & 22.3 \\
Fourth and more & 45 & 9.6 \\
& & \\
Gestation age (weeks) when attending ANCs* & & \\
for the first time & 88 & 18.7 \\
$4-15$ & 356 & 75.7 \\
$16-27$ & 26 & 5.5 \\
28 and above & & \\
\hline${ }^{*}$ Antenatal
\end{tabular}

$0.032)$ and marital status $(p=0.001)$ of the pregnant women (Table 2).

\section{Factors affecting implementation of IPT policy using SP}

The study explored a number of women related factors which could be affecting the implementation of IPT policy using SP, including attendance of pregnant women at the antenatal clinics. Almost two thirds 288 $(61.2 \%)$ of pregnant women started attending antenatal clinics after 20 weeks of gestation (Table 3).

During the day of interview, 179 (38.1\%) pregnant women were attending antenatal clinics for the second time (Table 3 ). The rest $141(30 \%)$ were attending antenatal clinics for the first time, $105(22.3 \%)$ for the third time and $45(9.6 \%)$ for the fourth or more times. There was an association between the level of knowledge about IPT policy using SP and the number of antenatal clinic visits by pregnant women $(p=0.041)$ (Table 4).

Pregnant women were asked if they had taken SP for IPT for their current pregnancies. It was reported that 220 (46.8 \%) pregnant women had not taken SP, 157 $(33.4 \%)$ had taken a single dose, $92(19.4$ $\%)$ had taken two doses and 1 (0.2 \%) reported to have been given three doses of SP when pregnant. The number of SP doses taken had an association with the level of knowledge of pregnant women for IPT policy using SP $(p<0.0001)$. Out of 220 pregnant women who had not been given SP during 
Table 4: Levels of knowledge of respondents about IPT policy using SP in relation to the number of antenatal clinic visits they had made $(\mathrm{N}=470)$.

\begin{tabular}{lcccc}
\hline \multirow{2}{*}{ Level of knowledge } & \multicolumn{4}{c}{ Visit } \\
\cline { 2 - 5 } & $\mathbf{1}^{\text {st }}$ & $\mathbf{2}^{\text {nd }}$ & $\mathbf{3}^{\text {rd }}$ & $\mathbf{4}^{\text {th }}$ \\
\hline Very low & $72(15 \%)$ & $86(18.3 \%)$ & $34(7.2 \%)$ & $18(3.8 \%)$ \\
Moderate & $37(7.9 \%)$ & $41(8.7 \%)$ & $28(6.0 \%)$ & $11(2.3 \%)$ \\
High & $32(6.8 \%)$ & $52(11.1 \%)$ & $43(9.1 \%)$ & $16(3.4 \%)$ \\
\hline Total & $\mathbf{1 4 1}(\mathbf{3 0 . 0} \%)$ & $\mathbf{1 7 9}(\mathbf{3 8 . 1} \%)$ & $\mathbf{1 0 5 ( 2 2 . 3 \% )}$ & $\mathbf{4 5 ( 9 . 6 )}$ \\
\hline
\end{tabular}

their current pregnancies, 214 (97.3\%) of them reported that the medication was out of stock when they attended antenatal clinics, 2 $(0.91 \%)$ refused to take the medication and 4 $(1.82 \%)$ did not attend the clinics on the day they were to be given the tablets. During the focus group discussion, a 45 years old pregnant woman at Utete district hospital said:

"Today I was supposed to take a dose of SP but when I came here I was told that the drug was out of stock. I was then told to buy the tablets from the neighboring pharmacy and then bring them to the clinic to be taken under direct observed therapy".

Pregnant women were asked to mention the measures they take when SP is out of stock at the antenatal clinics. Most (94.1\%) pregnant women reported that they do nothing, whereas 28 (5.9\%) said they would go to another health facility to look for SP if they are directed by the nurse. Other factors reported by pregnant women to be affecting the implementation of IPT policy include lack of bus fare to attend antenatal clinics (5.3\%) and unavailability of reliable transport to the health facilities $(1.9 \%)$.

In assessing acceptability of SP for IPT, pregnant women were asked to provide their views regarding SP-induced side effects and its tolerability. Two hundred and thirty one $(49.1 \%)$ pregnant women said that the tablets are well tolerated, while $222(47.2 \%)$ did not know any SP-induced side effects and could therefore not provide any response. It was only 16 (3.4\%) pregnant women who reported to have had experienced SPinduced side effects including rashes (12.5
$\%)$, fever (25\%), headache (12.5\%) and stomach cramps (50\%). During the focus group discussion, a middle-aged multigravidae woman at Ikwiriri mission reported:

"There were women who took SP on an empty stomach and started vomiting, they felt dizzy and became weak. Due to this problem, some women would not take SP, unless it is given under directly observed therapy at the health facility."

During the focus group discussions it was learnt that issues such as cost sharing, scarcity of health care providers, and long distances travelled to the health facilities are the main causes for poor attendance of pregnant women to antenatal clinics. As a result, this affects timely provision of SP for IPT to pregnant women. Family responsibilities including taking care of young children was commented to be another reason for pregnant women not attending antenatal clinics on time. In some health facilities pregnant women were required to go to the antenatal clinics with their spouses. This was seen to be a challenge since many husbands are usually not willing to escort their spouses to the clinics.

\section{The use of ALu as the drug of choice for treatment of malaria during pregnancy}

Out of 470 pregnant women who were interviewed, 43 (9.1\%) have had episode(s) of malaria fever during their current pregnancies. It was only $9(1.9 \%)$ pregnant women who reported to have used ALu for treatment of malaria. Out of 9 women who reported to have used ALu when pregnant, 3 
$(0.6 \%)$ were in the first trimester of pregnancy. All 470 women did not know the gestation period in which ALu is indicated for treatment of malaria in pregnant women. During focus group discussions, most women mentioned that $A L u$ is not safe to be used by pregnant women. This was echoed by a 27 year-old multigravidae participant at Ikwiriri mission, who said:

"I got malaria when I was 5 months pregnant, but I was told by the nurse that I cannot take ALu until the pregnancy was 7 months old. I was therefore given sulphamethoxyprazinepyrimethamine."

Only a small proportion (1.9\%) of pregnant women knew the correct dose and dosage regimen of $\mathrm{ALu}$. Among those who knew the correct dose and dosage regimen of ALu, only $8(1.7 \%)$ pregnant women knew that ALu should not be taken in an empty stomach. Two multigravidae participants who had used ALu when pregnant mentioned the dosage of ALu that were prescribed to them. One of them mentioned that she was told to take four tablets of ALu after every $8 \mathrm{~h}$ till she finishes 24 tablets. The other participant said that she was told to take four tablets at 2:00pm then another four tablets at 10:00pm, and then take the rest of the doses after every twelve hours for two consecutive days.

Quinine was mentioned by 202 (42.9\%) pregnant women as the most commonly used drug, followed by SP (23.8 \%), ALu (21.4 \%) and sulphamethoxyprazine-pyrimethamine) $(2.4 \%)$. Forty five $(9.5 \%)$ pregnant women could not remember the names of antimalarial drugs that they used when pregnant. In the focus group discussions it was reported that the commonest diseases that cause death to pregnant women in Rufiji were malaria, eclampsia, anaemia, post partum hemorrhage and heavy bleeding when pregnant. Most referral hospitals are far from where most of the residents reside, and therefore some deaths are mainly due to delays in referring patients to hospitals with qualified health care personnel and adequate facilities for diagnosis and treatment.

\section{DISCUSSION}

The results obtained show that almost half of the pregnant women had very low knowledge regarding the importance of IPT. Majority of pregnant women did not know why SP was provided, the correct timing of IPT and number of SP doses that are required for IPT during pregnancy. During focus group discussions it was evident that health care workers did not adequately sensitize and counsel pregnant women regarding the use of SP for IPT. These findings are similar to those previously reported by other researchers indicating that there is poor dissemination of information on the importance of IPT policy using SP among stakeholders including pregnant women in Tanzania [6].

The guidelines for maternal and child health stipulate that the first visit should be at the $16^{\text {th }}$ week, second visit at $20-24^{\text {th }}$, third visit at $28-32^{\text {nd }}$ week and the fourth visit at $36-40^{\text {th }}$ week of gestation [4]. In this study, about two-third of pregnant women started attending antenatal clinics at 16 weeks or more of gestation. Because of late and irregular attendance to antenatal clinics, a large number of pregnant women did not receive the recommended two doses of SP for IPT at the pre-determined times. A similar study that was conducted in Uganda in 2010 also indicated that late and irregular attendance to antenatal clinics disrupt antenatal schedules for proper delivery of IPT [9]. In the present study, pregnant women who had attended antenatal clinics for at least 3 times had better knowledge on IPT policy compared to those who had attended once or twice, suggesting an association between the number of antenatal visits and the level of knowledge on IPT policy among pregnant women.

It was also observed that there is an association between marital status, age and gravida of a woman and the level of knowledge on IPT policy. Majority of single pregnant women had very low knowledge 
compared to married ones. Results obtained from focus group discussions indicated that married women did get extra support from their spouses for attending antenatal clinics and subsequent use of SP for IPT. It was also observed that there has been increased sensitization at the antenatal clinics in most of the health facilities encouraging pregnant women to attend the clinics with their spouses so as to improve health education and support of pregnant women at the family level. This practice should be encouraged as it appears to have positive outcome on the overall health care given to pregnant women at the antenatal clinic and at home. The results also show that overall, older pregnant women had better understanding of IPT policy than the younger ones. In addition, multigravidae were more knowledgeable than primigravidae women regarding IPT. Similar to the findings of this study, other studies have also reported that age of the pregnant woman, time of registration with an antenatal provider, gravida and transport facilities available in the village were important predictors significantly affecting the utilization of minimum recommended antenatal care services $[9,10]$. These results indicate the need to have sensitization programs that that are designed to target different groups of pregnant women at the antenatal clinics.

For successful provision of IPT in pregnant women, it is imperative to ensure adequate and uninterrupted availability of SP at the health facilities [11, 12]. In this study, unavailability of SP was the main reason for about $50 \%$ of pregnant women not to have taken SP for IPT at the health facilities. These findings support the hypothesis that poor quality of healthcare services such as unavailability of medications and other basic services contribute to poor attendance of pregnant women at antenatal clinics [6].

Unless the benefits outweigh the risks, it is recommended that artemisinin should not be used in the first trimester of pregnancy because of its teratogenic effects including foetal resorption [13]. In the present study, about a quarter of pregnant women who were given ALu for treatment of malaria were in the first trimester of pregnancy. As shown by recent studies, the main reason for irrational use of antimalarial drugs in Tanzania is the lack of knowledge among health care providers $[14,15]$. It has also been reported that some pregnant women do not understand the instructions given by drug dispensers regarding the dosage and duration of use of ALu [3].

Lack of knowledge of antimalarial drug use during pregnancy is a serious problem, especially in areas of intense transmission such as Rufiji and other parts of Tanzania, where antimalarial drugs are given repeatedly to treat frequent fevers (even in the absence of malaria), thus increasing the risk of resistance and adverse drug reactions [14]. Due to lack of proper counseling of patients by health care providers about the benefits of ALu for the treatment of malaria, other pregnant women preferred using other antmalarial drugs such as sulphamethoxyprazine-pyrimethamine and SP which have been ruled out for the treatment of malaria in Tanzania. Unlike SP which was taken as a single dose for treatment of malaria, ALu is a multi-dose regimen taken over a period of three days. For many patients, this makes adherence a problem.

\section{Limitations of the study}

Participants in this study were pregnant women who were attending antenatal clinics. Due to the fact that some pregnant women may not be attending antenatal clinics, the overall knowledge and awareness of pregnant women regarding use of SP for IPT and ALu for treatment of malaria may be even much lower in Rufiji district. Due to the small sample size of both facilities and respondents in this study, the data on the knowledge and awareness about IPT and use of ALu among pregnant women reported here is limited in its generalizability beyond the study population. However, antenatal 
clinics were selected to represent a range of settings and levels of health facility, and sampling was done randomly in the district and therefore may have minimized source of bias. In addition, the national guidelines determining the timing of delivery of IPT and use of ALu for treatment of malaria apply throughout Tanzania. This suggests that the results presented here may apply elsewhere in Tanzania, especially in the rural areas, and therefore justify the need to conduct a larger survey to further investigate use of SP for IPT and rational use of antimalarial drugs during pregnancy.

\section{CONCLUSION}

Most pregnant women had below average level of knowledge about the use and benefits of SP for IPT and ALu for treatment of malaria during pregnancy. There is therefore a need to continuously sensitize and educate pregnant women about the use and benefits of antimalarial drugs. Sensitization programs should be designed to target different groups of pregnant women at the antenatal clinics and in the community.

\section{ACKNOWLEDGEMENT}

We acknowledge the financial support from SIDA-Sweden in collaboration with Muhimbili University of Health and Allied Sciences. We thank Dr. Peter Sasi, the district medical officer-in-charge in Rufiji for granting permission and support to conduct the study. We acknowledge the assistance of a social scientist, Dr. Edmund Kayombo for supervising focus group discussions. We extend our sincere thanks to the collaborators in the Reproductive Health Project, in particular Drs Dinah Gasarasi and Donati Tarimo for giving their time in reviewing the study protocol. We thank the nurses at the antenatal clinics for providing logistical support during interviews and focus group discussions.

\section{REFERENCES}

1. McGready $R$, Stepniewska $K$, Lindegardh $N$, Ashley EA, La Y, Singhasivanon $P$, White NJ, Nosten F. The pharmacokinetics of artemether and lumefantrine in pregnant women with uncomplicated falciparum malaria. Eur J Clin Pharmacol 2006; 62: 1021-1031.

2. Laffan S, James A, Maleeff B, Pagana J, Bushdid $P$, Clark R, White T. Mitochondrial involvement of artesunate toxicity in rat embryonic erythroblasts. Abstract: $46^{\text {th }}$ annual meeting of the Teratology Society; Tucson, Arizona, 2006.

3. Kamuhabwa AR, Mnyusiwalla F. Knowledge of drug dispensers and pregnant women on the use of artemether-lumefantrine during pregnancy. Tanzania J Health Res 2011; 13: 108-115.

4. World Health Organization guidelines for the treatment of malaria. Geneva: 2006.

5. Mubyazi $G$, Bloch $P$, Kamugisha $M$, Kitua $A$, ljumba J. Intermittent preventive treatment of malaria during pregnancy: a qualitative study of knowledge, attitudes and practices of district health managers, antenatal care staff and pregnant women in Korogwe District, NorthEastern Tanzania: Malar J 2005; 4: 31.

6. Anders $K$, Marchant $T$, Chambo $P$, Mapunda $P$, Reyburn H. Timing of intermittent preventive treatment for malaria during pregnancy and implications of current policy on early uptake in north-east Tanzania. Malar J 2008; 7: 79.

7. National Malaria Control Program (NMCP). Health Information Management Services and malaria reporting in Tanzania. Dar es Salaam, NMCP. $\mathrm{MoH} ; 2004$.

8. Ministry of Health and Social Welfare (MoH\&SW). National Guidelines for Malaria Diagnosis and Treatment. United Republic of Tanzania. Dar es Salaam; 2006.

9. Ndyomugyenyi $R$, Katamanywa J. Intermittent preventive treatment of malaria in pregnancy (IPTp): Do frequent antenatal care visits ensure access and compliance to IPTp in Ugandan rural communities: Trans $R$ Soc Trop Med Hyg 2010; 104: 536-540.

10. Khatib N, Zahiruddin QS, Gaidhane AM, Waghmare L, Srivatsava T, Goyal RC, Zodpey $S P$, Johrapurkar SR. Predictors for antenatal services and pregnancy outcome in a rural area: a prospective study in Wardha district, India. Indian J Med Sci 2009; 63: 436-444.

11. Hill J, Kazembe P. Reaching the Abuja target for intermittent preventive treatment of malaria in pregnancy in African women: a review of progress and operational challenges. Trop Med Int Health 2006; 1: 409-418.

12. Ndyomugyenyi $R$, Tukesiga $E$, Katamanywa $J$. Intermittent preventive treatment of malaria in pregnancy (IPTp): Participation of community directed distributors of ivermectin for onchocerciasis improves IPTp access in 
Ugandan rural communities. Trans $R$ Soc Trop Med Hyg 2009; 103:1221-1228.

13. Clark $R$, Kumemura $M$, Makori $N$, Nakata $Y$, Bernard F, Harrell A, White TEK, Arima A. Artesunate: Developmental Toxicity in Monkeys. Abstract, $46^{\text {th }}$ annual meeting of the Teratology Society; Tucson, Arizona, 2006.

14. Kamuhabwa A, Ramji K. Antimalarial Drugs for Pediatrics: Prescribing and Dispensing
Practices in Tanzanian City. Trop J Pharm Res 2011; 10: 611-618.

15. Guerin $P J$, Olliaro $P$, Nosten $F$, Druilhe $P$, Laxminarayan R, Binka F, Kilama WL, Ford $N$, White NJ. Malaria: current status of control, diagnosis, treatment, and a proposed agenda for research and development. Lancet Infect Dis 2002; 2: 564-573. 